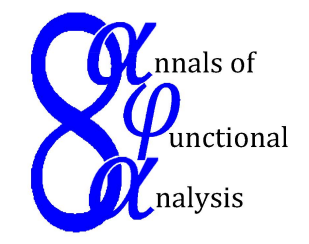

Ann. Funct. Anal. 6 (2015), no. 4, 60-69

http://doi.org/10.15352/afa/06-4-60

ISSN: 2008-8752 (electronic)

http://projecteuclid.org/afa

\title{
COMMON PROPERTIES OF THE OPERATOR PRODUCTS IN SPECTRAL THEORY
}

\author{
KAI YAN AND XIAOCHUN FANG* \\ Communicated by V. Müller
}

Abstract. Let $X, Y$ be Banach spaces, $A, D: X \rightarrow Y$ and $B, C: Y \rightarrow X$ be the bounded linear operators satisfying operator equation set

$$
\left\{\begin{array}{c}
A C D=D B D \\
D B A=A C A
\end{array} .\right.
$$

The concept of regularity was firstly introduced by Kordula and Müller. In this paper, we investigate the common properties of $A C$ and $B D$ in viewpoint of regularity when $A, B, C$ and $D$ all satisfy the operator equation set above.

\section{INTRODUCTION AND DEFINITIONS}

Throughout this paper, $\mathcal{B}(X, Y)$ denotes the set of all bounded linear operators from Banach space $X$ to Banach space $Y$. It is well known that if $A \in \mathcal{B}(X, Y)$ and $C \in \mathcal{B}(Y, X)$, then

$$
A C-I \text { is invertilbe } \Longleftrightarrow C A-I \text { is invertible, }
$$

see [[1], [6], [9], [11]]. This result is now known as Jacobson's lemma. In 2013, Corach, Duggal and Harte [[4]] generalized (1.1) and many of its relatives from $A C-I$ to certain $B A-I$ under the assumption

$$
A B A=A C A,
$$

where $A \in \mathcal{B}(X, Y)$ and $B, C \in \mathcal{B}(Y, X)$. They studied common properties of $A C$ and $B A$ in algebraic viewpoint and also obtained some nice topological analogues. In [[13]], Zeng and Zhong continued to investigate the common properties of $A C$

Date: Received: Nov. 6, 2014; Accepted: Mar. 10, 2015.

* Corresponding author.

2010 Mathematics Subject Classification. Primary 47A10; Secondary 47A53, 47A55.

Key words and phrases. Jacobson's lemma, operator equation set, common property, regularity. 
and $B A$ in the viewpoint of spectral theory. Recently, we gave a new extension of Jacobson's Lemma, in [[12]]: If $A, D \in \mathcal{B}(X, Y)$ and $B, C \in \mathcal{B}(Y, X)$ satisfy

$$
\left\{\begin{array}{c}
A C D=D B D \\
D B A=A C A
\end{array},\right.
$$

then

$$
A C-I \text { is invertible } \Longleftrightarrow B D-I \text { is invertible. }
$$

It is obviously that the case " $A=D$ ", which will give the equation (1.2), while the case

$$
\left\{\begin{array}{l}
A=D \\
B=C
\end{array},\right.
$$

which will give the Jacobson's lemma. In this note, we continue to study this situation and show that $A C$ and $B D$ share many common spectral properties.

We first fix some definitions in spectral theory. Throughout this paper, $\mathcal{B}(X)=$ $\mathcal{B}(X, X)$. For an operator $T \in \mathcal{B}(X)$, let $\mathcal{N}(T), \mathcal{R}(T)$ and $\sigma(T)$ denote the kernel, range and spectrum of $T$, respectively. The hyper-range of $T$ is the subspace $\mathcal{R}\left(T^{\infty}\right):=\bigcap \mathcal{R}\left(T^{n}\right)$, while the hyper-kernel of $T$ is the subspace $\mathcal{N}^{\infty}(T):=$ $\bigcup \mathcal{N}\left(T^{n}\right)$. In [[8]], Kordula and Müller gave the concept of regularity as follows:

Definition 1.1. A non empty subset $\mathbf{R} \subseteq \mathcal{B}(X)$ is called a regularity if it satisfies the following two conditions:

(1) If $A \in \mathcal{B}(X)$ and $n \geq 1$, then $A \in \mathbf{R}$ if and only if $A^{n} \in \mathbf{R}$.

(2) If $A, B, C, D \in \mathcal{B}$ are mutually commuting operators satisfying $A C+B D=$ $I$, then $A B \in \mathbf{R}$ if and only if $A, B \in \mathbf{R}$.

A regularity defines in a natural way the spectrum $\sigma_{\mathbf{R}}$ by $\sigma_{\mathbf{R}}(T)=\{\lambda \in \mathbb{C}$ : $\lambda I-T \notin \mathbf{R}\}$ for every $T \in \mathcal{B}(X)$. In [[8]], Kordula and Müller have proved that the spectrum $\sigma_{\mathbf{R}}$ corresponding to a regularity $\mathbf{R}$ satisfies the spectral mapping theorem - i.e., $f\left(\sigma_{\mathbf{R}}(T)\right)=\sigma_{\mathbf{R}}(f(T))$, for every $T \in B(X)$ and every analytic function $f$ on a neighborhood of $\sigma(T)$ which is non-constant on each component of its domain of definition.

For each $n \in \mathbb{N}:=\{0,1,2, \cdots\}$, we set

$$
c_{n}(T)=\operatorname{dim} \mathcal{R}\left(T^{n}\right) / \mathcal{R}\left(T^{n+1}\right), c_{n}^{\prime}(T)=\operatorname{dim} \mathcal{N}\left(T^{n+1}\right) / \mathcal{N}\left(T^{n}\right) .
$$

It is well known that, for every $n \in \mathbb{N}, c_{n}(T)=\operatorname{dim} X /\left(\mathcal{R}(T)+N\left(T^{n}\right)\right), c_{n}^{\prime}(T)=$ $\operatorname{dim} \mathcal{N}(T) \cap \mathcal{R}\left(T^{n}\right)$, see [[7], Lemma 3.2]. Hence, it is not hard to see that the sequences $\left\{c_{n}(T)\right\}_{n=0}^{\infty}$ and $\left\{c_{n}^{\prime}(T)\right\}_{n=0}^{\infty}$ are decreasing. For each $n \in \mathbb{N}, T$ induces a linear transformation from $\mathcal{R}\left(T^{n}\right) / \mathcal{R}\left(T^{n+1}\right)$ to $\mathcal{R}\left(T^{n+1}\right) / \mathcal{R}\left(T^{n+2}\right)$. Let $k_{n}(T)$ be the dimension of the null space of the induced map and let $k(T)=\sum_{n=0}^{\infty} k_{n}(T)$. From [[5], Lemma 2.3], it follows that

$$
\begin{aligned}
& k_{n}(T)=\operatorname{dim}\left(\mathcal{N}(T) \cap \mathcal{R}\left(T^{n}\right)\right) /\left(\mathcal{N}(T) \cap \mathcal{R}\left(T^{n+1}\right)\right) \\
& =\operatorname{dim}\left(\mathcal{R}(T)+\mathcal{N}\left(T^{n+1}\right)\right) /\left(\mathcal{R}(T)+\mathcal{N}\left(T^{n}\right)\right)
\end{aligned}
$$


for every $n \in \mathbb{N}$. We note that the sequence $\left\{k_{n}(T)\right\}_{n=0}^{\infty}$ is not always decreasing. From [[5], Theorem 3.7], it follows that

$$
\begin{aligned}
& k(T)=\operatorname{dim}\left(\mathcal{N}(T) /\left(\mathcal{N}(T) \cap \mathcal{R}\left(T^{\infty}\right)\right)\right. \\
& =\operatorname{dim}\left(\mathcal{R}(T)+\mathcal{N}\left(T^{\infty}\right)\right) / \mathcal{R}(T) .
\end{aligned}
$$

Similar to the definition of $k(T)$, we give the definitions of stable nullity and stable defect.

For an operator $T \in \mathcal{B}(X)$, the stable nullity $c^{\prime}(T)$ of $T$ is defined as

$$
c^{\prime}(T)=\sum_{n=0}^{\infty} c_{n}^{\prime}(T),
$$

while the stable defect $c(T)$ of $T$ is defined as

$$
c(T)=\sum_{n=0}^{\infty} c_{n}(T) .
$$

It is easy to see that $c(T)=\operatorname{dim} X / \mathcal{R}\left(T^{\infty}\right)$ and $c^{\prime}(T)=\operatorname{dim} \mathcal{N}\left(T^{\infty}\right)$.

Now, we give the definitions of some concrete subsets of regularity $\mathbf{R}_{\mathbf{i}} \subseteq B(X)$, $1 \leq i \leq 15$, which were introduced in [[10]], but in a different form.

Definition 1.2. $\mathbf{R}_{\mathbf{1}}=\{T \in \mathcal{B}(X): c(T)=0\}$,

$\mathbf{R}_{\mathbf{2}}=\{T \in \mathcal{B}(X): c(T)<\infty\}$,

$\mathbf{R}_{\mathbf{3}}=\left\{T \in \mathcal{B}(X)\right.$ : there exists $d \in \mathbb{N}$ such that $c_{d}(T)=0$ and $\mathcal{R}\left(T^{d+1}\right)$ is closed $\}$,

$\mathbf{R}_{\mathbf{4}}=\left\{T \in \mathcal{B}(X): c_{n}(T)<\infty\right.$ for every $\left.n \in \mathbb{N}\right\}$,

$\mathbf{R}_{\mathbf{5}}=\left\{T \in \mathcal{B}(X)\right.$ : there exists $d \in \mathbb{N}$ such that $c_{d}(T)<\infty$ and $\mathcal{R}\left(T^{d+1}\right)$ is closed\},

$\mathbf{R}_{\mathbf{6}}=\left\{T \in \mathcal{B}(X): c^{\prime}(T)=0\right.$ and $\mathcal{R}(T)$ is closed $\}$,

$\mathbf{R}_{\mathbf{7}}=\left\{T \in \mathcal{B}(X): c^{\prime}(T)<\infty\right.$ and $\mathcal{R}(T)$ is closed $\}$,

$\mathbf{R}_{\mathbf{8}}=\left\{T \in \mathcal{B}(X)\right.$ : there exists $d \in \mathbb{N}$ such that $c_{d}^{\prime}(T)=0$ and $\mathcal{R}\left(T^{d+1}\right)$ is closed\},

$\mathbf{R}_{\mathbf{9}}=\left\{T \in \mathcal{B}(X): c_{n}^{\prime}(T)<\infty\right.$ for every $n \in \mathbb{N}$ and $\mathcal{R}(T)$ is closed $\}$,

$\mathbf{R}_{10}=\left\{T \in \mathcal{B}(X)\right.$ : there exists $d \in \mathbb{N}$ such that $c_{d}^{\prime}(T)<\infty$ and $\mathcal{R}\left(T^{d+1}\right)$ is closed\},

$\mathbf{R}_{\mathbf{1 1}}=\{T \in \mathcal{B}(X): k(T)=0$ and $\mathcal{R}(T)$ is closed $\}$,

$\mathbf{R}_{\mathbf{1 2}}=\{T \in \mathcal{B}(X): k(T)<\infty$ and $\mathcal{R}(T)$ is closed $\}$,

$\mathbf{R}_{\mathbf{1 3}}=\left\{T \in \mathcal{B}(X)\right.$ : there exists $d \in \mathbb{N}$ such that $k_{n}(T)=0$ for every $n \geq d$ and $\mathcal{R}\left(T^{d+1}\right)$ is closed $\}$,

$\mathbf{R}_{\mathbf{1 4}}=\left\{T \in \mathcal{B}(X): k_{n}(T)<\infty\right.$ for every $n \in \mathbb{N}$ and $\mathcal{R}(T)$ is closed $\}$,

$\mathbf{R}_{\mathbf{1 5}}=\left\{T \in \mathcal{B}(X)\right.$ : there exists $d \in \mathbb{N}$ such that $k_{n}(T)<\infty$ for every $n \geq d$ and $\mathcal{R}\left(T^{d+1}\right)$ is closed $\}$.

Note that $\mathbf{R}_{1} \subseteq \mathbf{R}_{\mathbf{2}}=\mathbf{R}_{\mathbf{3}} \cap \mathbf{R}_{\mathbf{4}} \subseteq \mathbf{R}_{\mathbf{3}} \cup \mathbf{R}_{\mathbf{4}} \subseteq \mathbf{R}_{\mathbf{5}} \subseteq \mathbf{R}_{\mathbf{1 3}}, \mathbf{R}_{\mathbf{6}} \subseteq \mathbf{R}_{\mathbf{7}}=\mathbf{R}_{\mathbf{8}} \cap \mathbf{R}_{\mathbf{9}} \subseteq$ $\mathbf{R}_{\mathbf{3}} \cup \mathbf{R}_{\mathbf{4}} \subseteq \mathbf{R}_{10} \subseteq \mathbf{R}_{13}, \mathbf{R}_{11} \subseteq \mathbf{R}_{12}=\mathbf{R}_{13} \cap \mathbf{R}_{14} \subseteq \mathbf{R}_{13} \cup \mathbf{R}_{14} \subseteq \mathbf{R}_{15}$. Recently, Zeng and Zhong introduced several new regularities in [[14]]. We now give their definitions. 
Definition 1.3. $\mathbf{R}_{16}=\left\{T \in \mathcal{B}(X)\right.$ : there exists $d \in \mathbb{N}$ such that $c_{d}(T)=0$ and $\mathcal{R}(T)+\mathcal{N}\left(T^{d}\right)$ is closed $\}$,

$\mathbf{R}_{17}=\left\{T \in \mathcal{B}(X)\right.$ : there exists $d \in \mathbb{N}$ such that $c_{d}(T)<\infty$ and $\mathcal{R}(T)+\mathcal{N}\left(T^{d}\right)$ is closed $\}$,

$\mathbf{R}_{18}=\left\{T \in \mathcal{B}(X)\right.$ : there exists $d \in \mathbb{N}$ such that $k_{n}(T)=0$ for every $n \geq d$ and $\mathcal{R}(T)+\mathcal{N}\left(T^{d}\right)$ is closed $\}$,

$\mathbf{R}_{19}=\left\{T \in \mathcal{B}(X)\right.$ : there exists $d \in \mathbb{N}$ such that $k_{n}(T)<\infty$ for every $n \geq d$ and $\mathcal{R}(T)+\mathcal{N}\left(T^{d}\right)$ is closed $\}$.

The operators of $\mathbf{R}_{\mathbf{1}}, \mathbf{R}_{\mathbf{2}}, \mathbf{R}_{\mathbf{3}}, \mathbf{R}_{\mathbf{4}}$ and $\mathbf{R}_{\mathbf{5}}$ are surjective, lower semi-Browder, right Drazin invertible, lower semi-Fredholm and right essentially Drazin invertible, respectively, while the operators of $\mathbf{R}_{\mathbf{6}}, \mathbf{R}_{\mathbf{7}}, \mathbf{R}_{\mathbf{8}}, \mathbf{R}_{\mathbf{9}}$ and $\mathbf{R}_{10}$ are bounded below, upper semi-Browder, left Drazin invertible, upper semi-Fredholm and left essentially Drazin invertible operators, respectively. The operators of $\mathbf{R}_{\mathbf{1 1}}, \mathbf{R}_{\mathbf{1 2}}$ and $\mathbf{R}_{13}$ are semi-regular, essentially semi-regular and quasi-Fredholm operators, respectively. The operators of $\mathbf{R}_{18}$ are called the operators with eventual topological uniform descent. We refer readers to [[5], [10]] for more definitions and details.

In this note, we establish that if $A, D \in \mathcal{B}(X, Y)$ and $B, C \in \mathcal{B}(Y, X)$ satisfy

$$
\left\{\begin{array}{l}
A C D=D B D \\
D B A=A C A
\end{array},\right.
$$

then

$$
B D-I \in \mathbf{R}_{i} \Longleftrightarrow A C-I \in \mathbf{R}_{i}, 1 \leq i \leq 19 .
$$

It extends the main result (Theorem 2.8) of [[13]] from the special case " $A=D$ " to the general case. Thus, many results in [[14]] become the very special case of our results. Moreover, it perfects the spectral results in part 2 of [[12]].

\section{Common properties For $A C$ AND $B D$}

In this section, we consider the elementary components of regularities $\mathbf{R}_{\mathbf{i}}(1 \leq$ $i \leq 19$ ). Let us begin with the following lemma, which will give the basic relations between kernel, or range, of $A C$ and that of $B D$.

Lemma 2.1. If $A, D \in \mathcal{B}(X, Y), B, C \in \mathcal{B}(Y, X)$ satisfy $\left\{\begin{array}{c}A C D=D B D \\ D B A=A C A\end{array}\right.$ and $f$ is a polynomial, then we have

(1) $D \mathcal{R}(f(B D-I)) \subseteq \mathcal{R}(f(A C-I))$;

(2) $D \mathcal{N}(f(B D-I)) \subseteq \mathcal{N}(f(A C-I))$;

(3) $B A C \mathcal{R}(f(A C-I)) \subseteq \mathcal{R}(f(B D-I))$;

(4) $B A C \mathcal{N}(f(A C-I)) \subseteq \mathcal{N}(f(B D-I))$.

Proof. (1) Let $x \in \mathcal{R}(f(B D-I))$ - i.e., there exists $x_{0} \in X$ such that $x=$ $f(B D-I) x_{0}$. Thus, by $A C D=D B D$, we have

$$
D x=D\left(f(B D-I) x_{0}\right)=f(A C-I) D x_{0} \in \mathcal{R}(f(A C-I)) .
$$

This implies $D \mathcal{R}(f(B D-I)) \subseteq \mathcal{R}(f(A C-I))$. 
(2) Let $x \in \mathcal{N}(f(B D-I))$. Then, by $A C D=D B D$, we have

$$
f(A C-I) D x=D f(B D-I) x=0 .
$$

This implies $D \mathcal{N}(f(B D-I)) \subseteq \mathcal{N}(f(A C-I))$.

(3) Let $y \in \mathcal{R}(f(A C-I))$ - i.e., there exists $y_{0} \in Y$ such that $y=f(A C-I) y_{0}$. Thus, by $D B A=A C A$, we have

$$
B A C y=B A C f(A C-I) y_{0}=f(B D-I) B A C y_{0} \in \mathcal{R}(f(B D-I)) .
$$

This implies $B A C \mathcal{N}(f(A C-I)) \subseteq \mathcal{N}(f(B D-I))$.

(4) Let $y \in \mathcal{N}(f(A C-I))$. Then, by $D B A=A C A$, we have

$$
f(B D-I) B A C y=B A C f(A C-I) y=0 .
$$

This implies $B A C \mathcal{N}(f(A C-I)) \subseteq \mathcal{N}(f(B D-I))$.

We observe the following lemma by the symmetry immediately.

Lemma 2.2. If $A, D \in \mathcal{B}(X, Y), B, C \in \mathcal{B}(Y, X)$ satisfy $\left\{\begin{array}{c}A C D=D B D \\ D B A=A C A\end{array}\right.$ and $f$ is a polynomial, then we have

(1) $A \mathcal{R}(f(C A-I)) \subseteq \mathcal{R}(f(D B-I))$;

(2) $A \mathcal{N}(f(C A-I)) \subseteq \mathcal{N}(f(D B-I))$;

(3) $C D B \mathcal{R}(f(D B-I)) \subseteq \mathcal{R}(f(C A-I))$;

(4) $C D B \mathcal{N}(f(D B-I)) \subseteq \mathcal{N}(f(C A-I))$;

The Lemma 2.1 is crucial for the following Lemmas 2.3-2.5, while in the proofs of Lemmas 2.3-2.5, we only use the polynomial $f(x)=(x-1)^{n}$ or $f(x)=$ $(x-1)^{n+1}$. The proof of the following Lemmas 2.3-2.5 are inspired by the very special case

$$
\left\{\begin{array}{l}
A=D \\
B=C
\end{array}\right.
$$

which have been proved recently in [[14]] by Zeng and Zhong.

Lemma 2.3. If $A, D \in \mathcal{B}(X, Y)$ and $B, C \in \mathcal{B}(Y, X)$ satisfy $\left\{\begin{array}{l}A C D=D B D \\ D B A=A C A\end{array}\right.$, then $c_{n}^{\prime}(A C-I)=c_{n}^{\prime}(B D-I)$ for all $n \in \mathbb{N}$. Consequently, $c^{\prime}(A C-I)=$ $c^{\prime}(B D-I)$.

Proof. In order to prove the dimension of $\mathcal{N}\left((B D-I)^{n+1}\right) / \mathcal{N}\left((B D-I)^{n}\right)$ is equal to that of $\mathcal{N}\left((A C-I)^{n+1}\right) / \mathcal{N}\left((A C-I)^{n}\right)$, we define a linear map $\hat{D}\left(c_{n}^{\prime}\right)$ induced by $D$ from

$$
\mathcal{N}\left((B D-I)^{n+1}\right) / \mathcal{N}\left((B D-I)^{n}\right)
$$

to

$$
\mathcal{N}\left((A C-I)^{n+1}\right) / \mathcal{N}\left((A C-I)^{n}\right) .
$$

Since (2) of Lemma 2.1 implies $D \mathcal{N}\left((B D-I)^{n}\right) \subseteq \mathcal{N}\left((A C-I)^{n}\right)$, we have that $\hat{D}\left(c_{n}^{\prime}\right)$ is well defined. 
Now, we claim that $\hat{D}\left(c_{n}^{\prime}\right)$ is injective. Indeed, let $x \in \mathcal{N}\left((B D-I)^{n+1}\right)$ and $D x \in \mathcal{N}\left((A C-I)^{n}\right)$. Thus, we have $B A C D x \in \mathcal{N}\left((B D-I)^{n}\right)$ by (4) of Lemma 2.3. Therefore,

$$
\begin{aligned}
x & =B D x-(B D-I) x \\
& =B D x-B A C D x+B A C D x-(B D-I) x \\
& =B D x-B D B D x+B A C D x-(B D-I) x \\
& =B A C D x-(B D+I)(B D-I) x \\
& \in \mathcal{N}\left((B D-I)^{n}\right) .
\end{aligned}
$$

This implies $\hat{D}\left(c_{n}^{\prime}\right)$ is injective, as desire.

Thus,

$$
\begin{aligned}
c_{n}^{\prime}(B D-I) & \leq c_{n}^{\prime}(A C-I) \\
& =c_{n}^{\prime}(C A-I) \text { by [[14], Lemma 3.10] } \\
& \leq c_{n}^{\prime}(D B-I) \text { by the symmetry } \\
& =c_{n}^{\prime}(B D-I) \text { by [[14], Lemma 3.10]. }
\end{aligned}
$$

So $c_{n}^{\prime}(A C-I)=c_{n}^{\prime}(B D-I)$ for all $n \in \mathbb{N}$.

Lemma 2.4. If $A, D \in \mathcal{B}(X, Y)$ and $B, C \in \mathcal{B}(Y, X)$ satisfy $\left\{\begin{array}{l}A C D=D B D \\ D B A=A C A\end{array}\right.$, then $c_{n}(A C-I)=c_{n}(B D-I)$ for all $n \in \mathbb{N}$. Consequently, $c(A C-I)=$ $c(B D-I)$.

Proof. In order to prove the dimension of $\mathcal{R}\left((B D-I)^{n}\right) / \mathcal{R}\left((B D-I)^{n+1}\right)$ is equal to that of $\mathcal{R}\left((A C-I)^{n}\right) / \mathcal{R}\left((A C-I)^{n+1}\right)$, we define a linear map $\hat{D}\left(c_{n}\right)$ induced by $D$ from

$$
\mathcal{R}\left((B D-I)^{n}\right) / \mathcal{R}\left((B D-I)^{n+1}\right)
$$

to

$$
\mathcal{R}\left((A C-I)^{n}\right) / \mathcal{R}\left((A C-I)^{n+1}\right) .
$$

Since (1) of Lemma 2.1 implies that

$$
D\left(\mathcal{R}\left((B D-I)^{n+1}\right)\right) \subseteq \mathcal{R}\left((A C-I)^{n+1}\right),
$$

we obtain that $\hat{D}\left(c_{n}\right)$ is well defined.

Now, we claim that $\hat{D}\left(c_{n}\right)$ is injective. Indeed, let $x \in \mathcal{R}\left((B D-I)^{n}\right)$ and $D x \in \mathcal{R}\left((A C-I)^{n+1}\right)$. Thus, we have $B A C D x \in \mathcal{R}\left((B D-I)^{n+1}\right)$ by (3) of Lemma 2.1. Therefore, by the similar computation in the proof of lemma 2.3, we have

$$
\begin{aligned}
x & =B D x-(B D-I) x \\
& =B D x-B A C D x+B A C D x-(B D-I) x \\
& =B D x-B D B D x+B A C D x-(B D-I) x \\
& =B A C D x-(B D+I)(B D-I) x \\
& \in \mathcal{R}\left((B D-I)^{n+1}\right) .
\end{aligned}
$$


This implies $\hat{D}\left(c_{n}\right)$ is injective, as desire.

Therefore,

$$
\begin{aligned}
c_{n}(B D-I) & \leq c_{n}(A C-I) \\
& =c_{n}(C A-I) \text { by [[14], Lemma 3.9] } \\
& \leq c_{n}(D B-I) \text { by the symmetry } \\
& =c_{n}^{\prime}(B D-I) \text { by [[14], Lemma 3.9]. }
\end{aligned}
$$

So $c_{n}(A C-I)=c_{n}(B D-I)$ for all $n \in \mathbb{N}$.

Lemma 2.5. If $A, D \in \mathcal{B}(X, Y)$ and $B, C \in \mathcal{B}(Y, X)$ satisfy $\left\{\begin{array}{c}A C D=D B D \\ D B A=A C A\end{array}\right.$, then $k_{n}(A C-I)=k_{n}(B D-I)$ for all $n \in \mathbb{N}$. Consequently, $k(A C-I)=$ $k(B D-I)$.

Proof. In order to prove the dimension of $\left(\mathcal{R}(B D-I)+\mathcal{N}\left((B D-I)^{n+1}\right)\right) /(\mathcal{R}(B D-$ $\left.I)+\mathcal{N}\left((B D-I)^{n}\right)\right)$ is equal to that of $\left(\mathcal{R}(A C-I)+\mathcal{N}\left((A C-I)^{n+1}\right)\right) /(\mathcal{R}(A C-$ $\left.I)+\mathcal{N}\left((A C-I)^{n}\right)\right)$, we define a linear map $\hat{D}\left(k_{n}\right)$ induced by $D$ from

$$
\left(\mathcal{R}(B D-I)+\mathcal{N}\left((B D-I)^{n+1}\right)\right) /\left(\mathcal{R}(B D-I)+\mathcal{N}\left((B D-I)^{n}\right)\right)
$$

to

$$
\left(\mathcal{R}(A C-I)+\mathcal{N}\left((A C-I)^{n+1}\right)\right) /\left(\mathcal{R}(A C-I)+\mathcal{N}\left((A C-I)^{n}\right)\right) .
$$

Since (1) and (2) of Lemma 2.1 implies that

$$
D\left(\mathcal{R}(B D-I)+\mathcal{N}\left((B D-I)^{n+1}\right)\right) \subseteq \mathcal{R}(A C-I)+\mathcal{N}\left((A C-I)^{n+1}\right),
$$

we obtain that $\hat{D}\left(k_{n}\right)$ is well defined.

Now, we claim that $\hat{D}\left(k_{n}\right)$ is injective. Indeed, let $x \in \mathcal{R}(B D-I)+\mathcal{N}((B D-$ $\left.I)^{n+1}\right)$ and $D x \in \mathcal{R}(A C-I)+\mathcal{N}\left((A C-I)^{n}\right)$. Then, there exist $y \in Y$ and $z \in \mathcal{N}\left((A C-I)^{n}\right)$ such that $D x=(A C-I) y+z$. and hence $y=A C y+z-D x$. Thus, by

$$
\left\{\begin{array}{c}
A C D=D B D \\
D B A=A C A
\end{array},\right.
$$

we have

$$
\begin{aligned}
x & =B D x-(B D-I) x \\
& =B[(A C-I) y+z]-(B D-I) x \\
& =B(A C-I)(A C y+z-D x)+B z-(B D-I) x \\
& =B A C A C y-B A C y+B A C z-B z-B A C D x+B D x+B z-(B D-I) x \\
& =B D B A C y-B A C y+B A C z-B D B D x+B D x-(B D-I) x \\
& =(B D-I)(B A C y-B D x-x)+B A C z .
\end{aligned}
$$

Since $z \in \mathcal{N}\left((A C-I)^{n}\right)$, we obtain $x \in \mathcal{R}(B D-I)+\mathcal{N}\left((B D-I)^{n}\right)$ by (4) of Lemma 2.1. This implies that $\hat{D}\left(k_{n}\right)$ is injective, as desire. 
Similar to Proposition 2.3 and Proposition 2.4, we have

$$
\begin{aligned}
k_{n}(B D-I) & \leq k_{n}(A C-I) \\
& =k_{n}(C A-I) \text { by }[[14], \text { Lemma } 3.8] \\
& \leq k_{n}(D B-I) \text { by the symmetry } \\
& =k_{n}^{\prime}(B D-I) \text { by [[14], Lemma 3.8]. }
\end{aligned}
$$

So $k_{n}(A C-I)=k_{n}(B D-I)$ for all $n \in \mathbb{N}$.

Lemma 2.6. If $A, D \in \mathcal{B}(X, Y)$ and $B, C \in \mathcal{B}(Y, X)$ satisfy $\left\{\begin{array}{c}A C D=D B D \\ D B A=A C A\end{array}\right.$, then for all $n \in \mathbb{N}, \mathcal{R}(A C-I)+\mathcal{N}\left((A C-I)^{n}\right)$ is closed if and only if $\mathcal{R}(B D-$ $I)+\mathcal{R}\left((B D-I)^{n}\right)$ is closed.

Proof. Suppose that $\mathcal{R}(A C-I)+\mathcal{N}\left((A C-I)^{n}\right)$ is closed. Let $\left\{x_{n}\right\}_{n=1}^{\infty} \subseteq \mathcal{R}(B D-$ $I)+\mathcal{N}\left((B D-I)^{n}\right)$ be a sequence such that $x_{n} \rightarrow x$. Then, for each positive integer $n$, there exist $y_{n} \in \mathcal{R}(B D-I)$ and $z_{n} \in \mathcal{N}\left((B D-I)^{n}\right)$ such that $x_{n}=$ $y_{n}+z_{n}$. Hence, $D x=\lim _{n \rightarrow \infty} D x_{n}=\lim _{n \rightarrow \infty} D\left(y_{n}+z_{n}\right)$. From (1) and (2) of Lemma 2.1, we have $D y_{n} \in \mathcal{R}(A C-I)$ and $D z_{n} \in \mathcal{N}\left((A C-I)^{n}\right)$. Since $\mathcal{R}(A C-I)+\mathcal{N}\left((A C-I)^{n}\right)$ is closed, there exist $y \in Y$ and $z \in \mathcal{N}\left((A C-I)^{n}\right)$ such that $D x=(A C-I) y+z$ and hence $y=A C y+z-D x$. Thus, by the similar computation in the proof of lemma 2.5 , we have

$$
\begin{aligned}
x & =B D x-(B D-I) x \\
& =B[(A C-I) y+z]-(B D-I) x \\
& =B(A C-I)(A C y+z-D x)+B z-(B D-I) x \\
& =B A C A C y-B A C y+B A C z-B z-B A C D x+B D x+B z-(B D-I) x \\
& =B D B A C y-B A C y+B A C z-B D B D x+B D x-(B D-I) x \\
& =(B D-I)(B A C y-B D x-x)+B A C z .
\end{aligned}
$$

Since $z \in \mathcal{N}\left((A C-I)^{n}\right)$, we obtain $x \in \mathcal{R}(B D-I)+\mathcal{N}\left((B D-I)^{n}\right)$ by (4) of Lemma 2.1. This implies $\mathcal{R}(B D-I)+\mathcal{N}\left((B D-I)^{n}\right)$ is closed.

To the opposite implication, suppose that $\mathcal{R}(B D-I)+\mathcal{N}\left((B D-I)^{n}\right)$ is closed. Then we have

$$
\begin{aligned}
& \mathcal{R}(B D-I)+\mathcal{N}\left((B D-I)^{n}\right) \text { is closed } \\
& \Leftrightarrow \mathcal{R}(D B-I)+\mathcal{N}\left((D B-I)^{n}\right) \text { is closed ( by [[14], Lemma 3.11]) } \\
& \Rightarrow \mathcal{R}(C A-I)+\mathcal{N}\left((C A-I)^{n}\right) \text { is closed ( by the symmetry) } \\
& \Leftrightarrow \mathcal{R}(A C-I)+\mathcal{N}\left((A C-I)^{n}\right) \text { is closed ( by [[14], Lemma 3.11]). }
\end{aligned}
$$

Therefore, the conclusion hold.

Next, we obtain a corollary which is a special case " $n=0$ " of Lemma 2.6.

Corollary 2.7. If $A, D \in \mathcal{B}(X, Y)$ and $B, C \in \mathcal{B}(Y, X)$ satisfy $\left\{\begin{array}{l}A C D=D B D \\ D B A=A C A\end{array}\right.$ , then $\mathcal{R}(A C-I)$ is closed if and only if $\mathcal{R}(B D-I)$ is closed. 
Corollary 2.8. If $A, D \in \mathcal{B}(X, Y)$ and $B, C \in \mathcal{B}(Y, X)$ satisfy $\left\{\begin{array}{l}A C D=D B D \\ D B A=A C A\end{array}\right.$ , then, for all $n \in \mathbb{N}, \mathcal{R}(A C-I)^{n}$ is closed if and only if $\mathcal{R}(B D-I)^{n}$ is closed. Proof. For an arbitrary $n \in \mathbb{N}$, take

$$
B_{n}=\sum_{k=1}^{n}\left(\begin{array}{c}
n \\
k
\end{array}\right)(-1)^{k}(B D)^{k-1} B
$$

and

$$
C_{n}=\sum_{k=1}^{n}\left(\begin{array}{c}
n \\
k
\end{array}\right)(-1)^{k} C(A C)^{k-1} .
$$

Then, it is not hard to check the following equations

$$
\begin{gathered}
\left\{\begin{aligned}
A C_{n} D & =D B_{n} D \\
D B_{n} A & =A C_{n} A,
\end{aligned}\right. \\
(I-B D)^{n}=I-B_{n} D
\end{gathered}
$$

and

$$
(I-A C)^{n}=I-A C_{n} .
$$

Thus, from Corollary 2.7, we obtain that $\mathcal{R}(A C-I)^{n}$ is closed if and only if $\mathcal{R}(B D-I)^{n}$ is closed for all $n \in \mathbb{N}$.

\section{Conclusion}

Now, all the elementary components of regularities $\mathbf{R}_{\mathbf{i}}(1 \leq i \leq 19)$ have been considered in Lemma 2.3, 2.4, 2.5, 2.6 and Corollary 2.7, 2.8, then we obtain the following result (Theorem 3.1), which extends the main result (Theorem 2.8) in [[13]] from the special case " $A=D$ " to the general case. Moreover, it is known that $\mathbf{R}_{\mathbf{1}}$ and $\mathbf{R}_{\mathbf{6}}$ are the surjective and bounded below operators, respectively. Hence, the Proposition 2.2 and 2.5 in [[12]] are contained in the following theorem.

Theorem 3.1. If $A, D \in \mathcal{B}(X, Y)$ and $B, C \in \mathcal{B}(Y, X)$ satisfy $\left\{\begin{array}{c}A C D=D B D \\ D B A=A C A\end{array}\right.$, then

$$
\sigma_{\boldsymbol{R}_{i}}(A C) \backslash\{0\}=\sigma_{\boldsymbol{R}_{i}}(B D) \backslash\{0\} \text { for } 1 \leq i \leq 19 .
$$

Proof. From the Lemma 2.3, 2.4, 2.5, 2.6 and the Corollary 2.7, 2.8, the conclusion follows immediately.

We conclude this note by an example to illustrate that the results obtained in this note are proper generalizations of the corresponding ones in [[13]].

Example 3.2. For Banach spaces $X$ and $Y$, let $S_{1} \in \mathcal{B}(X, X), S_{2}, T_{1} \in \mathcal{B}(Y, X)$ and $T_{2} \in \mathcal{B}(X, Y)$ be arbitrary nonzero operators satisfying $S_{1}=S_{2} T_{2}$ and $T_{2} T_{1} \neq I$. We consider $A, B, C \in \mathcal{B}(X \oplus Y)$ as follows respectively:

$$
A=\left(\begin{array}{cc}
0 & S_{2} \\
0 & 0
\end{array}\right), B=\left(\begin{array}{cc}
S_{1} & 0 \\
0 & 0
\end{array}\right), C=D=\left(\begin{array}{cc}
I & T_{1} \\
T_{2} & I
\end{array}\right) .
$$


It is easy to check that

$$
C A C-C B C=\left(\begin{array}{cc}
0 & S_{2}\left(I-T_{2} T_{1}\right) \\
0 & T_{2} S_{2}\left(I-T_{2} T_{1}\right)
\end{array}\right) \neq 0
$$

but,

and

$$
A C B=B C B=\left(\begin{array}{cc}
S_{1}^{2} & 0 \\
0 & 0
\end{array}\right)
$$

$$
B C A=A C A=\left(\begin{array}{cc}
0 & S_{1} S_{2} \\
0 & 0
\end{array}\right) .
$$

Hence, the common spectral properties, or regularity, of $A C$ and $C B$ can only be deduced directly from the results obtained in this note, but not from the corresponding ones in [[13]].

Acknowledgement. This work has been supported by National Natural Science Foundation of China (11371279).

\section{REFERENCES}

1. B.A. Barnes, Common operator properties of the linear operators $R S$ and $S R$, Proc. Amer. Math. Soc. 126 (1998), no. 4. 1055-1061.

2. C. Benhida and E.H. Zerouali, Local Spectral Theory of Linear Operators RS and SR, Integral Equations Operator Theory 54 (2006), no. 1, 1-8.

3. M. Berkani, Restriction of an operator to the range of its powers, Studia Math. 140 (2000), no. 2, 163-175.

4. G. Corach, B. Duggal and R. Harte, Extensions of Jacobson's lemma, Comm. Algebra 41 (2013), no. 2, 520-531.

5. S. Grabiner, Uniform ascent and descent of bounded operators, J. Math. Soc. Japan 34 (1982), no. 2, 317-337.

6. R. Harte, Spectral Mapping Theorems A Bluffer's Guide, Springer, 2014.

7. M.A. Kaashoek, Ascent, descent, nullity and defect, a note on a paper by A. E. Taylor, Math. Ann. 172 (1967), no. 2, 105-115.

8. V. Kordula and V. Müller, On the axiomatic theory of spectrum, Studia Math. 119 (1996), no. 2. 109-128.

9. C. Lin, Z. Yan and Y. Ruan, Common properties of operators RS and $S R$ and p-hyponormal operators, Integral Equations Operator Theory 43 (2002), no. 3. 313-325.

10. M. Mbekhta and V. Müller, On the axiomatic theory II, Studia Math. 119 (1996), no. 2. 129-147.

11. V. Müller, Spectral Theory of Linear Operators and Spectral Systems in Banach Algebras, second edition, Birkhäuser, Basel, Boston, Berlin, 2007.

12. K. Yan and X.C. Fang, Common properties of the operator products in local spectral theory, submitted.

13. Q.P. Zeng and H.J. Zhong, Common properties of bounded linear operators $A C$ and BA: Spectral theory, Math. Nachr. 267 (2014), no. 5-6. 717-725.

14. Q.P. Zeng and H.J. Zhong, New results on common properties of the bounded linear operators $R S$ and $S R$, Acta Math. Sinica (English Series) 29 (2013), no. 10. 1871-1884.

Department of Mathematics, Tongui University, Shanghai 200092, P.R. China. E-mail address: yklolxj@163.com

E-mail address: xfang@tongji.edu.cn 